\title{
Development of a Smooth Trajectory Maneuver Method to Accommodate the Ares I Flight Control Constraints
}

\author{
Robin M. Pinson, ${ }^{1}$ Terri L. Schmitt ${ }^{2}$ and John M. Hanson ${ }^{3}$ \\ NASA Marshall Space Flight Center, MSFC,AL,35812
}

\begin{abstract}
Six degree-of-freedom (DOF) launch vehicle trajectories are designed to follow an optimized 3-DOF reference trajectory. A vehicle has a finite amount of control power that it can allocate to performing maneuvers. Therefore, the 3-DOF trajectory must be designed to refrain from using $100 \%$ of the allowable control capability to perform maneuvers, saving control power for handling off-nominal conditions, wind gusts and other perturbations. During the Ares I trajectory analysis, two maneuvers were found to be hard for the control system to implement; a roll maneuver prior to the gravity turn and an angle of attack maneuver immediately after the $\mathrm{J}$-2X engine start-up. It was decided to develop an approach for creating smooth maneuvers in the optimized reference trajectories that accounts for the thrust available from the engines. A feature of this method is that no additional angular velocity in the direction of the maneuver has been added to the vehicle after the maneuver completion. This paper discusses the equations behind these new maneuvers and their implementation into the Ares I trajectory design cycle. Also discussed is a possible extension to adjusting closed-loop guidance.
\end{abstract}

\section{Nomenclature}

$\begin{array}{ll}\alpha & =\text { angular acceleration } \\ c_{l s} & =\text { acceleration integration coefficient prior to switch } \\ c_{2 s} & =\text { velocity integration coefficient prior to switch } \\ c_{l e} & =\text { acceleration integration coefficient after the switch } \\ c_{2 e} & =\text { velocity integration coefficient after the switch } \\ \theta_{s} & =\text { angle and angular rates (its derivatives) prior to switch } \\ \theta_{e} & =\text { angle and angular rates (its derivatives) after the switch } \\ \theta_{s w} & =\text { angle and angular rates (its derivatives) at the switch } \\ t_{s} & =\text { maneuver start time } \\ t_{e} & =\text { maneuver end time } \\ t_{s w} & =\text { maneuver switch time }\end{array}$

\section{Introduction}

$I_{\text {six }}$

$\mathrm{N}$ order to allow a new launch vehicle design to fly efficiently and in a stable manner, the controls experts and six degree-of-freedom (DOF) simulation programmers need smooth trajectories that the flight control system can accommodate; while saving sufficient margin to manage perturbations and wind gusts. Sudden changes in vehicle orientation are unrealistic and challenge the vehicle's control system. If the flight control system is asked to perform maneuvers in a sudden manner the system will attempt to do so, even with the high probability of a hard-over gimbal situation. Flying with actuators in the hard-over position jeopardizes the vehicle stability by removing any ability to counteract perturbations and wind gusts. Three degree-of-freedom (translational motion modeled in three dimensions with the attitude perfectly aligned in the desired direction) trajectory simulations may allow instantaneous angle and angular velocity changes, which are not feasible for a control system. Without any guidelines, the 3-DOF trajectory designers must estimate the imposed vehicle limits or leave the angular velocity unconstrained. To avoid these situations, new smoother maneuvers were developed based on a vehicle's available

\footnotetext{
${ }^{1}$ Aerospace Engineer, Guidance, Navigation and Mission Analysis, MSFC AL 35812 / EV42, and AIAA Member

${ }^{2}$ Aerospace Engineer, Guidance, Navigation and Mission Analysis, MSFC AL 35812 / EV42, and AIAA Member

${ }^{3}$ Aerospace Engineer, Flight Mechanics and Analysis, MSFC AL 35812 / EV40, and AIAA Member
} 
thrust. These maneuvers are a more realistic characterization of what the vehicle is capable of flying. Two different maneuvers were studied with this new method; a pure body roll and an angle of attack change. These maneuvers were designed to assist in the Ares I analysis efforts.

Launch vehicles typically fly an open-loop phase - pre-determined attitude profile as a function of an independent variable, such as time - through the atmosphere. Next a closed-loop phase - feedback analysis based on the current navigated state - follows during the near vacuum ascent to orbit. Space Shuttle heritage provides the Powered Explicit Guidance (PEG) algorithm as a way to determine the closed-loop trajectory. This method is a linear tangent steering law based on the tangent of the thrust attitude angle. PEG determines the velocity vector to be gained from the thrust, in order to reach the desired end state via a predictor-corrector method. The results from PEG are sent to a steering algorithm that uses a second order control law, along with vehicle parameters, to determine the commands sent to the vehicle. Shuttle open-loop guidance follows a set of pre-determined attitudes as a function of velocity. Additional details on the Shuttle closed-loop guidance calculations are presented in Ref. 1.

The high fidelity simulation, Marshall Aerospace Vehicle Representation in C (MAVERIC), used to analyze the new Ares I designs, is based on this Shuttle heritage and other similar methods. In order to develop the trajectories for MAVERIC, a reference optimized trajectory is first developed by using the Program to Optimize Simulated Trajectories (POST). The POST trajectory is a 3-DOF open-loop optimized trajectory based on the vehicle's configuration. Unlike the MAVERIC simulation and the Shuttle simulations, the POST trajectory does not contain any closed-loop flight segments. In order to keep the simulations coupled without significant differences between them, the portion of the POST trajectory that provides the open-loop attitude for the MAVERIC open-loop portion cannot tax the flight control system. The segment that corresponds to the closed-loop section needs to fly similarly or the optimization will not be representative of what will be seen in the closed-loop segments in the MAVERIC simulation.

Two maneuvers in the initial Ares I POST design were discovered to have sudden changes in orientation that were unrealistic and would challenge the vehicle's control capability. When designing these two maneuvers, POST was instructed to optimize the corresponding angular rate without imposing limits. Therefore, the optimized rates tended to be unrealistic. Thus, the MAVERIC and POST simulations were becoming detached and causing a situation which needed attention. Programming the PEG algorithm into an optimizer would only assist in designing maneuvers within the closedloop flight segments, hence only one of the two maneuvers.

The maneuvers that were singled out as candidates for this new method were a roll maneuver (during open-loop) and an angle of attack maneuver (during closed-loop). Figure 1 contains the relative yaw, pitch, and roll angles, along with the angle of attack profile during a reference

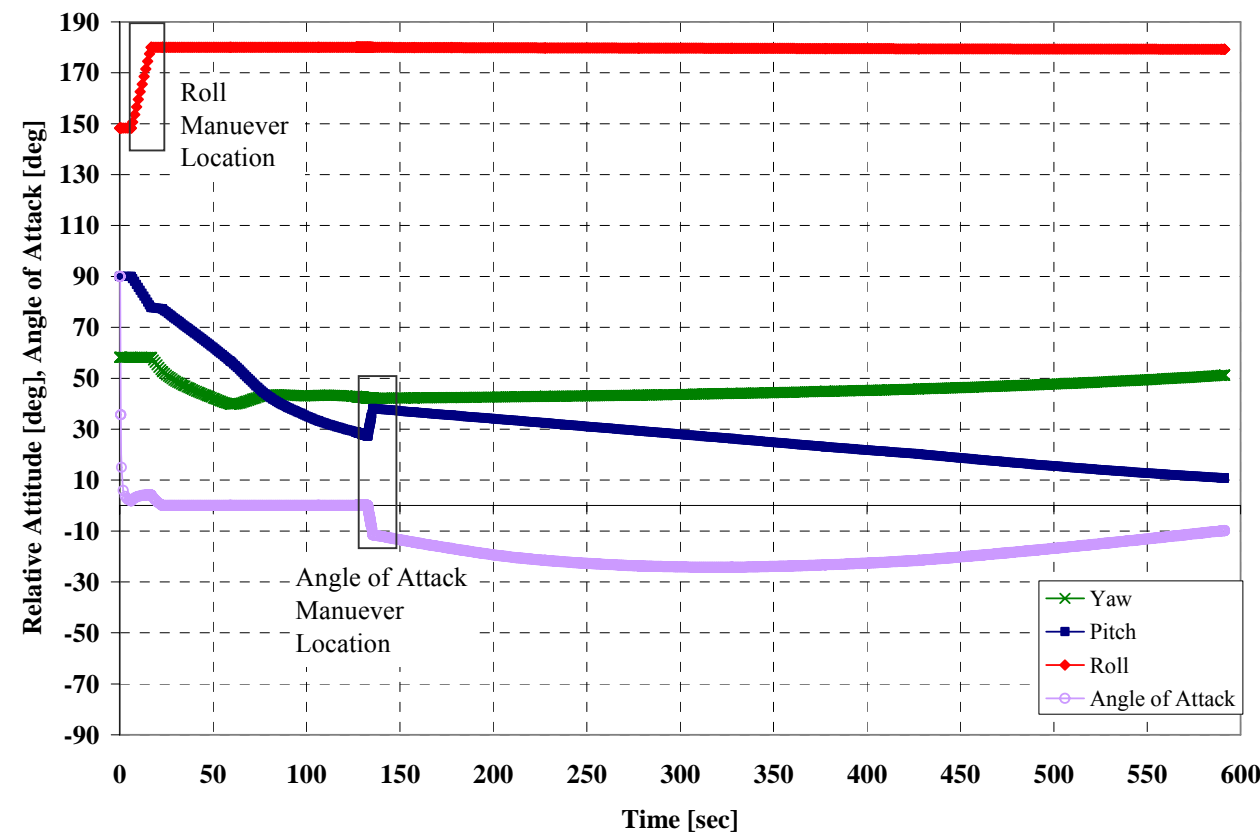

Figure 1. Angle of Attack, Relative Yaw, Pitch and Roll for a POST Trajectory without the Smoother Method. Ares I trajectory.

The locations for the roll and angle of attack maneuvers are outlined on the plot. These two areas correspond to the largest change in angle per elapsed time. The smoother method discussed in this paper was developed to more realistically fly these two maneuvers to allow a better transition between the trajectory design software and the flight 
simulation. These equations can work for any launch vehicle design, though they were originally done for the NASA Ares I launch vehicle.

\section{Roll Maneuver Calculations}

Due to the Ares I orientation on the pad, the vehicle must roll into the desired azimuth unless the launch azimuth is exactly $90.0^{\circ}$. In addition, the roll is designed to maintain the required heads-down orientation of the crew. On the launch pad, the crew are oriented with their heads pointing east. Since the ISS mission has a higher inclination (51.6 deg) than the lunar mission ( $28.5 \mathrm{deg})$, its larger total roll angle has been the primary focus. This roll maneuver occurs after the Ares I clears the launch tower, but prior to the start of the gravity turn. The gravity turn is the portion of flight where the trajectory is designed with zero angle of attack and zero sideslip angle (flying into the relative wind), in order to minimize structural bending loads.

The goal of adding a roll maneuver is to create a smoother trajectory and follow a more realistic path that the Reaction Control System (RCS) thrusters and the thrust vector control system can accommodate while allowing sufficient margin. In the previous studies, the angular rate was optimized with no imposed limits for the roll maneuver. In Figure 2 the green curve corresponds to this older method. This roll rate was $3.0 \mathrm{deg} / \mathrm{s}$ and instantaneous changes to and from $0.0 \mathrm{deg} / \mathrm{s}$ were permitted. After some analysis, this roll rate was believed to be excessive for the control system to handle, especially transitioning into and out of the roll maneuver. The blue line in Figure 2 corresponds to this newer smoother method for the exact same case. Use of a smooth profile minimizes reaction control system (roll control) propellant usage and overshoot, as well as providing margin for the system to handle other unwanted roll torques. The overshoot is a problem since the flight control system will not know that a stop at 180 degrees is desired, until it is reached and then passed at a $3.0 \mathrm{deg} / \mathrm{s}$ rate. Since the attitude acceleration is limited by the RCS thrust, significant overshoot occurs before the system recovers.

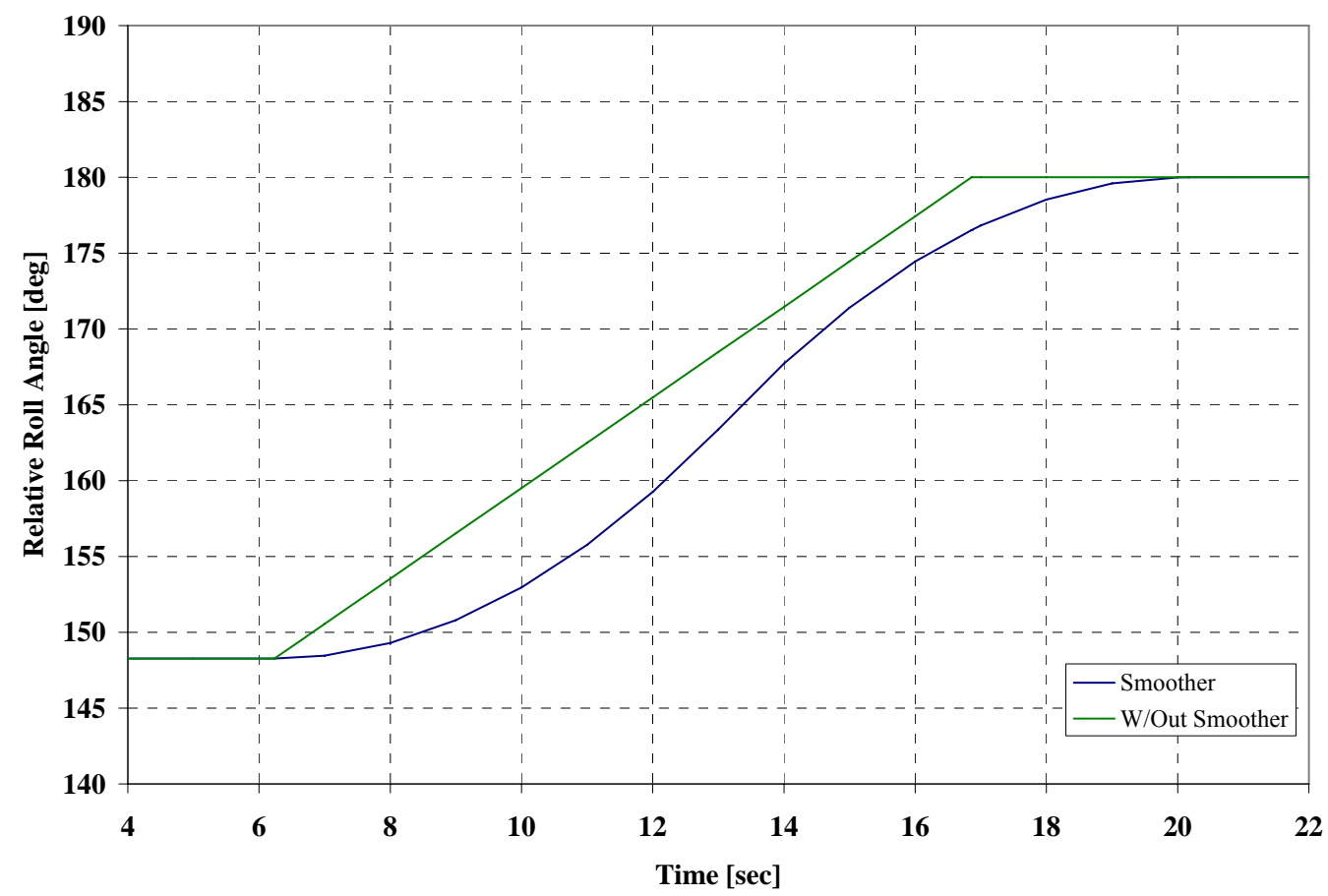

Figure 2. Roll with Smoother and with Optimized Rate.

The Ares I design has four RCS thrusters allotted for roll control. In order to allow margin for the control system to handle contingencies, only half of the available RCS thrust was allocated for the roll maneuver. It was assumed that the RCS thrust available was a constant value and that the thrust vector can change $180^{\circ}$ in direction instantaneously. These assumptions made the equations easier to work with and allowed the excess angular velocity added to the system for the maneuver to be completely removed prior to the vehicle achieving its new roll orientation.

Available angular acceleration from the RCS thrusters was calculated from Eq. (1). 


$$
\ddot{\theta}=\frac{T_{R C S} d}{I_{\text {roll }}}=\alpha
$$

$T_{R C S}$ is half of the thrust from the RCS thrusters, with $d$ as the moment arm - the distance from the vehicle centerline to the thrusters - and $I_{\text {roll }}$ is the moment of inertia around the roll (X) axis. It was assumed that all three of these values are constant during the maneuver, even though the inertia will change with time. Since it is a constant angular acceleration, $\alpha$, the angular velocity and the roll angle equations can be determined by integrating the angular acceleration, Eq. (2) and Eq. (3). The $c_{i}$ are integration constants that are currently unknown, but can be determined from initial conditions.

$$
\begin{gathered}
\dot{\theta}=\alpha t+c_{1} \\
\theta=\frac{1}{2} \alpha t^{2}+c_{1} t+c_{2}
\end{gathered}
$$

Approximately halfway through the maneuver, the angular acceleration vector will switch directions. The time that this occurs will be referred to as the switch time. In the case of the Ares I, the roll maneuver is around the positive $\mathrm{X}$ axis, so the first half of the maneuver has a positive angular acceleration and the second half has a negative angular acceleration. This created four equations for calculating the smoother angle curve. The integration coefficients before the switch point are different than the coefficients after the switch point, in addition to different signs of $\alpha$. This created a system of four equations, Eq. (4), which had to be solved for the four integration coefficients, the switch time, and the maneuver end time. The subscript $s$ refers to the variables corresponding to the start of the maneuver (prior to the switch) and $e$ to the variables after the switch.

$$
\begin{aligned}
& \dot{\theta}_{s}=\alpha t+c_{1 s} \\
& \theta_{s}=\frac{1}{2} \alpha t^{2}+c_{1 s} t+c_{2 s} \\
& \dot{\theta}_{e}=-\alpha t+c_{1 e} \\
& \theta_{e}=-\frac{1}{2} \alpha t^{2}+c_{1 e} t+c_{2 e}
\end{aligned}
$$

Prior to calculating the unknown constants, the following conditions are known: the maneuver start time, the initial roll angle, the final roll angle, and the initial and final angular velocities. An assumption was made that at the switch point the angular velocity and the roll angle must be continuous. These conditions were sufficient to solve for the unknown coefficients and times. (5).

From the start time, the initial angle and the initial angular velocity, $c_{1 s}$ and $c_{2 s}$, can be easily calculated as in Eq.

$$
\begin{aligned}
& c_{1 s}=\dot{\theta}_{s}-\alpha t_{s} \\
& c_{2 s}=\theta_{s}-\frac{1}{2} \alpha t_{s}^{2}-c_{1 s} t_{s}
\end{aligned}
$$

With the above values calculated, six more equations, located in Eq. (6), can be formed to aid in solving for the unknowns. Since the switch point is a critical point and has requirements of continuity associated with it, the angle and angular rate at the switch point from both sets of coefficients are determined. The abbreviation $s w$ refers to the values occurring at the switch point. 


$$
\begin{aligned}
& \dot{\theta}_{s w}=\alpha t_{s w}+c_{1 s} \\
& \theta_{s w}=\frac{1}{2} \alpha t_{s w}{ }^{2}+c_{1 s} t_{s w}+c_{2 s} \\
& \dot{\theta}_{s w}=-\alpha t_{s w}+c_{1 e} \\
& \theta_{s w}=-\frac{1}{2} \alpha t_{s w}{ }^{2}+c_{1 e} t_{s w}+c_{2 e} \\
& \dot{\theta}_{e}=-\alpha t_{e}+c_{1 e} \\
& \theta_{e}=-\frac{1}{2} \alpha t_{e}{ }^{2}+c_{1 e} t_{e}+c_{2 e}
\end{aligned}
$$

Impose the continuity constraint by setting the angle and angular velocity occurring at the switch time from the two sets of equations equal to one another. This allows Eq. (6) to be reduced to the set of four equations in Eq. (7).

$$
\begin{aligned}
& c_{1 e}=2 \alpha t_{s w}+c_{1 s} \\
& c_{2 e}=\alpha t_{s w}^{2}+\left(c_{1 s}-c_{1 e}\right) t_{s w}+c_{2 s} \\
& \dot{\theta}_{e}=-\alpha t_{e}+c_{1 e} \\
& \theta_{e}=-\frac{1}{2} \alpha t_{e}{ }^{2}+c_{1 e} t_{e}+c_{2 e}
\end{aligned}
$$

The equations in Eq. (7) can be reduced to a system of two equations which are functions of only switch time and end time.

$$
\begin{aligned}
& 2 \alpha t_{s w}-\dot{\theta}_{e}-\alpha t_{e}+c_{1 s}=0 \\
& \alpha t_{s w}^{2}-t_{s w}\left(-c_{1 s}+\dot{\theta}_{e}+\alpha t_{e}\right)-\theta_{e}-\frac{1}{2} \alpha t_{e}^{2}+\dot{\theta}_{e} t_{e}+\alpha t_{e}^{2}+c_{2 s}=0
\end{aligned}
$$

Solving this system of equations located in Eq. (8) with a symbolic mathematical solver provides a valid switch and end time for the given parameters. Since the system has quadratic equations, there will be two results from the mathematical solver for the time. A quick check will reveal the solution that is real and valid based on the known start time. With these values, the remaining unknown constants $c_{l e}$ and $c_{2 e}$ can be calculated. With all the coefficients in the equations known, along with the switch and end times, a table of time versus roll angle can be computed.

\section{Ares I Application of Roll Maneuver}

This method was applied to the Ares I initial trajectory optimization runs during Design Analysis Cycle II. By using Matlab as the mathematical solver for the equations listed in the above section, the roll table was created and then incorporated into the POST trajectory optimization process. The angular acceleration used in the equations was from half of the available RCS thrust, creating a roll maneuver around the body X axis.

During the design cycles, POST is the first simulation to work with the newest vehicle design models. The optimized trajectories created by POST are the basis for the MAVERIC 3-DOF and 6-DOF simulations. These MAVERIC simulations are critical for the variety of Guidance, Navigation and Control analyses that the vehicle must undergo during design.

The Euler angle guidance schemes in POST are based on either the inertial launch site coordinate system or a geographic relative coordinate system. Using the inertial Euler angles, it is not possible to input a pure maneuver around the body axes (the table produced from the equations is a pure body rotation). However, it is possible to setup the initial orientation of the vehicle in the simulation to achieve a roll maneuver around the body axes with the 
geographic relative coordinate system. In POST, the relative Euler angles sequence is yaw, pitch, roll. The yaw angle is defined as the azimuth angle of the body X axis measured from North to East; pitch is the elevation of the body $\mathrm{X}$ axis above the local horizontal, and roll is the angle about the body $\mathrm{X}$ axis. The relative angle sequence must orient the vehicle on the pad correctly in the inertial frame while allowing the vehicle to have the astronauts' heads down when the relative roll is $180^{\circ}$. In order to do this, the initial relative sequence sets the yaw angle to the launch azimuth value and the pitch angle to $90^{\circ}$. The roll angle is initially defined as the sum of the launch azimuth plus $90^{\circ}$. This sequence of POST's relative Euler angles will allow a pure body roll from a commanded roll angle. Detailed descriptions of POST, including the coordinate systems, are located in Ref. 2.

POST allows the launch azimuth to vary as an independent variable. Since the launch azimuth determines the initial roll angle, it affects the initial conditions for the roll maneuver. This prevents prior calculation of the single curve that the maneuver needs to follow. Therefore, a family of curves was developed as an input into POST. To cover the needs for monthly variations and launch window analyses, a set of curves was formed that covers the initial launch azimuth from $30^{\circ}$ to $70^{\circ}$ for the ISS mission, with a curve for every degree. The final angle at the end of the maneuver remains at $180.0^{\circ}$ for every curve. This allows POST to take the current launch azimuth (roll angle) and select the appropriate curve. Since the curves are spaced close together, interpolation between the two closest curves is possible without greatly changing the required RCS thrust to produce the maneuver.

The initial roll angle for the lunar mission is close to $180^{\circ}$, so the range in the table is for launch azimuth of 70 degrees to $89.99^{\circ}\left(90^{\circ}\right.$ launch azimuth corresponds to $180^{\circ}$ initial roll.) Due to the launch window analysis, additional data was constructed for select lunar cases because the launch azimuth becomes greater than $90^{\circ}$. A range of curves was added from $90.01^{\circ}$ to $110^{\circ}$. In order to calculate the curves that have an initial azimuth greater than $90^{\circ}$, the angular acceleration was negative prior to the switch point and positive after the switch. In the equations for calculating the curves $\alpha$ is a negative value.

For Ares I, the vehicle configuration for both the lunar and ISS missions are similar. So, only one set of vehicle parameters was needed to create the families of roll curves. These calculations require knowledge of the 6-DOF mass properties. The data in Table 1 are the parameters needed to calculate the curves. The data (Table 1) used as an example for the roll calculation in this paper is from a vehicle similar to an Ares I vehicle.

An example of a family of curves for the ISS cases is located in Figure 3. The graph shows a curve for every degree from an initial launch azimuth of $40^{\circ}$ through an initial launch azimuth of $70^{\circ}$, using the data in Table 1 .

Table 1. Roll Maneuver Calculation Inputs.

\begin{tabular}{|l|l|}
\hline Thrust & $2 * 780 \mathrm{lbf}=1560 \mathrm{lbf}$ \\
\hline Moment arm $(\mathrm{d})$ & $9.0 \mathrm{ft}$ \\
\hline $\mathrm{I}_{\mathrm{roll}}\left(\mathrm{I}_{\mathrm{xx}}\right)$ & $1,218,764 \mathrm{slug}-\mathrm{ft}^{2}$ \\
\hline Initial Angle & $35^{\circ}-70^{\circ}\left(1.0^{\circ}\right.$ increments $)$ \\
& $70^{\circ}-89.99^{\circ} ;$ \\
& $90.01^{\circ}-100^{\circ}$ degrees $\left(1.0^{\circ}\right.$ increments $)$ \\
\hline Initial Angular Velocity & $0.0 \mathrm{deg} / \mathrm{sec}$ \\
\hline Final Angle & $180.0^{\circ}$ \\
\hline Final Angular Velocity & $0.0 \mathrm{deg} / \mathrm{sec}$ \\
\hline
\end{tabular}




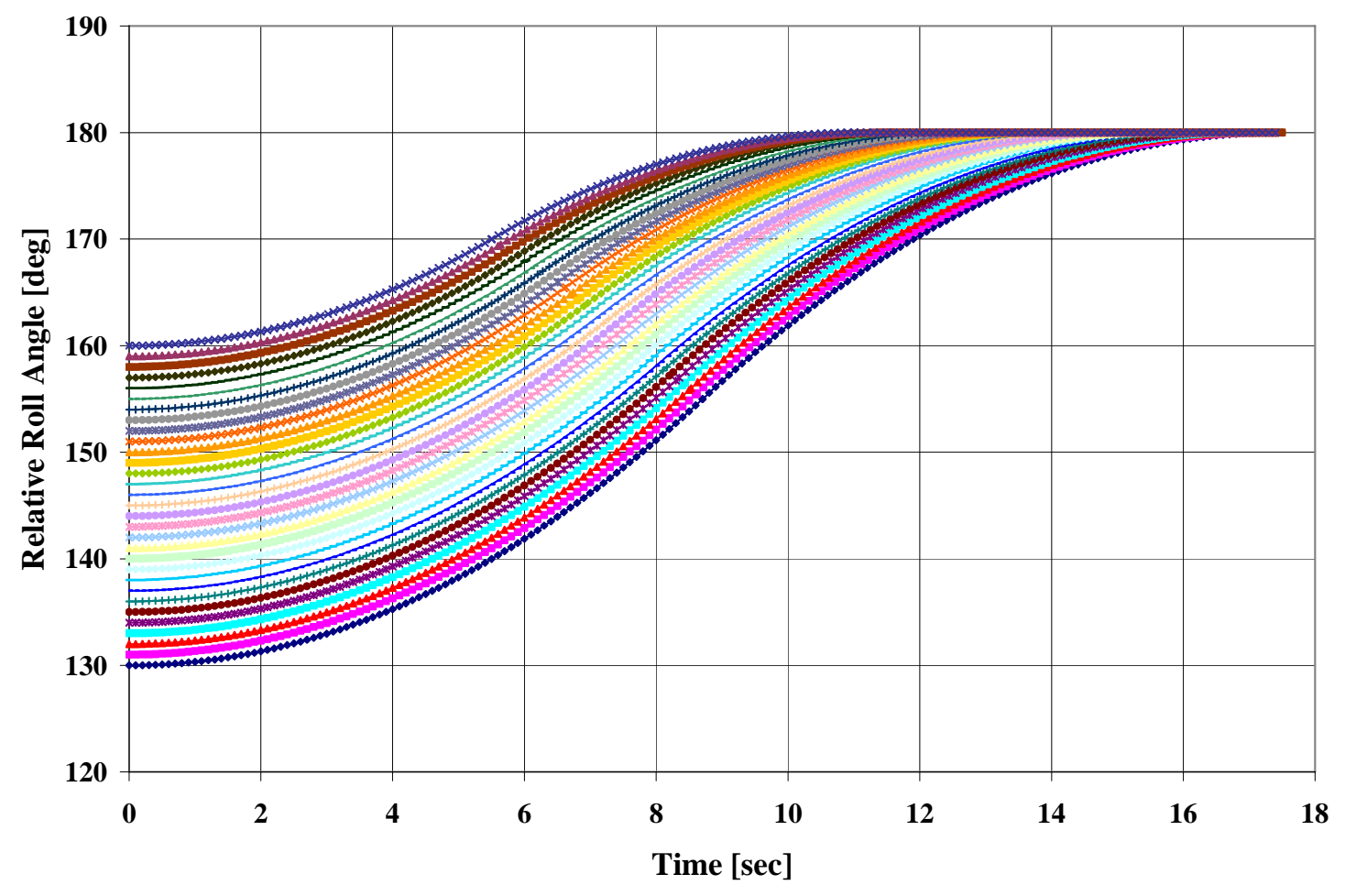

Figure 3. Roll Maneuver Curves for ISS Mission: Initial Launch Azimuth $\mathbf{4 0}^{\circ}-\mathbf{7 0}^{\circ}$.

Similarly, an example of a family of curves for the lunar cases is in Figure 4. The graph shows a curve for every degree from an initial launch azimuth of $75^{\circ}$ through an initial launch azimuth of $100^{\circ}$.

As the vehicle design cycle progresses, the parameters are updated to reflect vehicle changes. New curves are subsequently produced for the POST input deck. In addition the range of initial launch azimuth angles was adjusted to $37^{\circ}$ to $114,^{\circ}$ based on the Eastern Test Range's acceptable limits. 


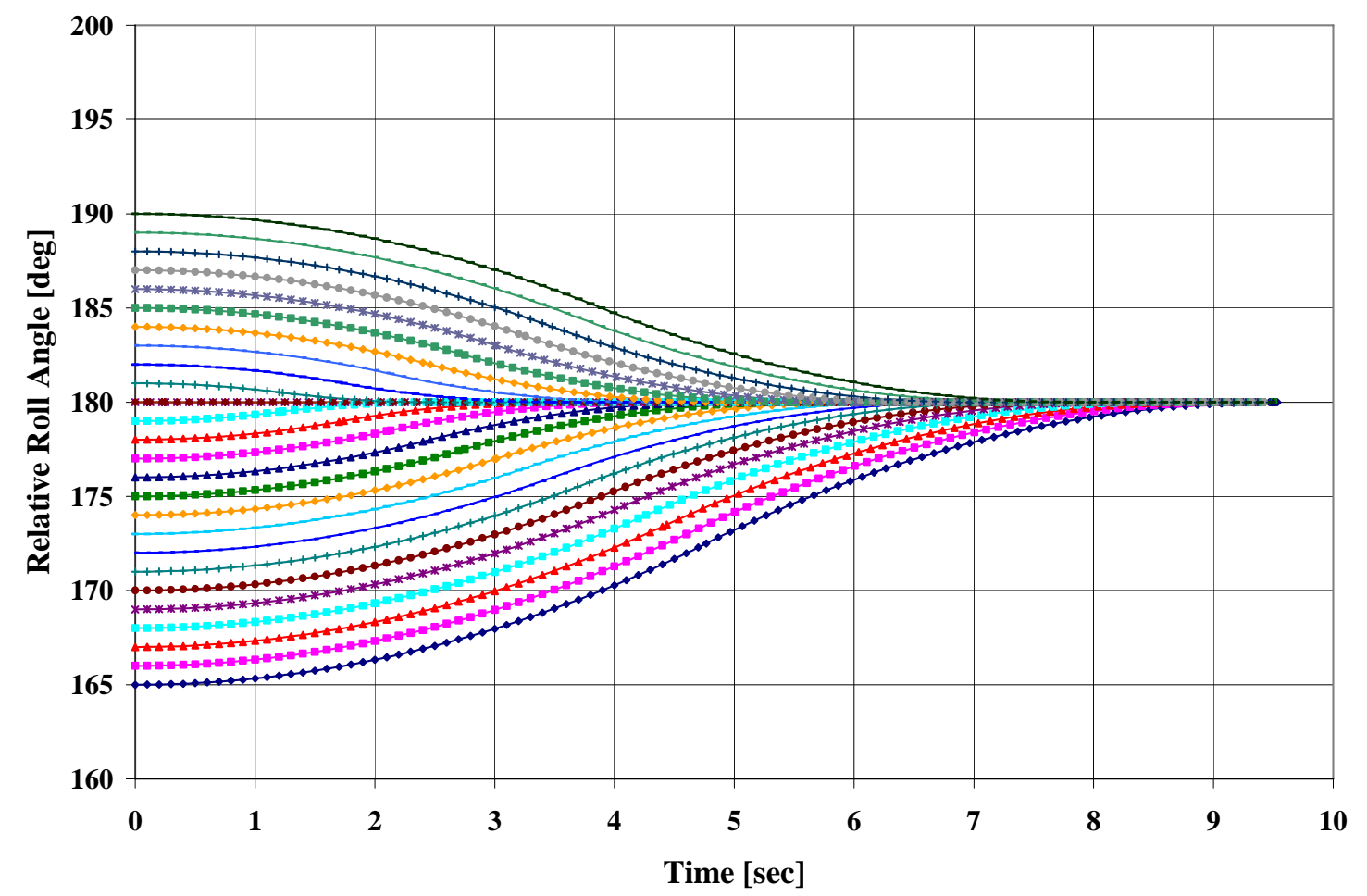

Figure 4. Roll Maneuver Curves for Lunar Mission: Initial Launch Azimuth $75^{\circ}-100^{\circ}$.

\section{Angle of Attack Maneuver}

Earlier trajectory revisions in POST had a second maneuver that was also difficult for the control system to perform. This maneuver occurs immediately after the J-2X engine reached its $100 \%$ thrust level. It occurs here because the vehicle has completed its gravity turn and must maneuver to the optimum attitude for the remainder of the flight to orbit. The maneuver rotates from a zero degree angle of attack to an optimized angle of attack that POST calculates. The following sections of the trajectory fly with an optimized inertial pitch profile.

In Figure 5, the green curve represents a case that POST ran without the smoother applied. The optimizer had determined that the best angle of attack rate should be $4.0 \mathrm{deg} / \mathrm{sec}$. The transition from $0.0 \mathrm{deg} / \mathrm{s}$ to $4.0 \mathrm{deg} / \mathrm{s} \mathrm{was}$ instantaneous, as was the transition back down to $0.0 \mathrm{deg} / \mathrm{sec}$ after the maneuver. Overshoot will occur when the desired rate transitions to zero instantaneously at the end of the maneuver. In terms of the implementation in a 6DOF simulation, the POST result will not reflect how the guidance and control system actually fly, resulting in differing results between the trajectory design and the simulation. The blue curve is from the same case with the smoother applied, which does not have instantaneous rate transitions. At the end of the smooth maneuver the angle of attack rate decreases until it reaches $0.0 \mathrm{deg} / \mathrm{s}$ at completion. The curves in Figure 5 include results from the beginning of the flight segment immediately following the angle of attack maneuver segment. The next segment also includes a small change in angle of attack, which appears in the plot. The curves that POST interpolated from for the smooth angle of attack maneuver (blue curve), had a zero angle of attack rate at the end of the curve, though this is not apparent in Figure 5. Without a smooth command that reserves a portion of the control authority, the thrust vector control actuators will saturate, resulting in a loss of vehicle stability.

For the Ares I POST trajectories, a maneuver using aerodynamic angles was inserted prior to resuming the optimized inertial pitch profile. The calculation method described in Eq. (2) - Eq. (8) can be easily modified to produce a smooth angle of attack maneuver. This will avoid using a large angle of attack rate to reach the desired angle at the beginning of the optimized inertial pitch profile.

In this case, the J-2X engine was used instead of the RCS thrusters to maneuver the vehicle. The J-2X engine gimbals for both pitch and yaw control. It has the capability to gimbal $4.0^{\circ}$, however only $1.5^{\circ}$ was allowed for the nominal maneuver calculation. This reserves the remaining gimbal motion to correct contingencies encountered in 
the pitch and yaw directions. In order to use the previous equations, the angular acceleration for this maneuver must be calculated, Eq. (9).

$$
\ddot{\theta}=\frac{L T \sin \delta}{I_{\text {pitch }}}=\alpha
$$

$L$ is the moment arm or the distance from the vehicle center of mass to the gimbal point; with $I_{\text {pitch }}$ as the moment of inertia around the pitch axis (body $\mathrm{Y}$ axis) and $\delta$ is the gimbal angle. $T$ is the thrust produced by the $\mathrm{J}-2 \mathrm{X}$ engine. Currently, the angle of attack maneuver occurs after the J-2X engine has finished the start-up profile and has reached its steady-state condition. Therefore, the thrust and angular acceleration are constant.

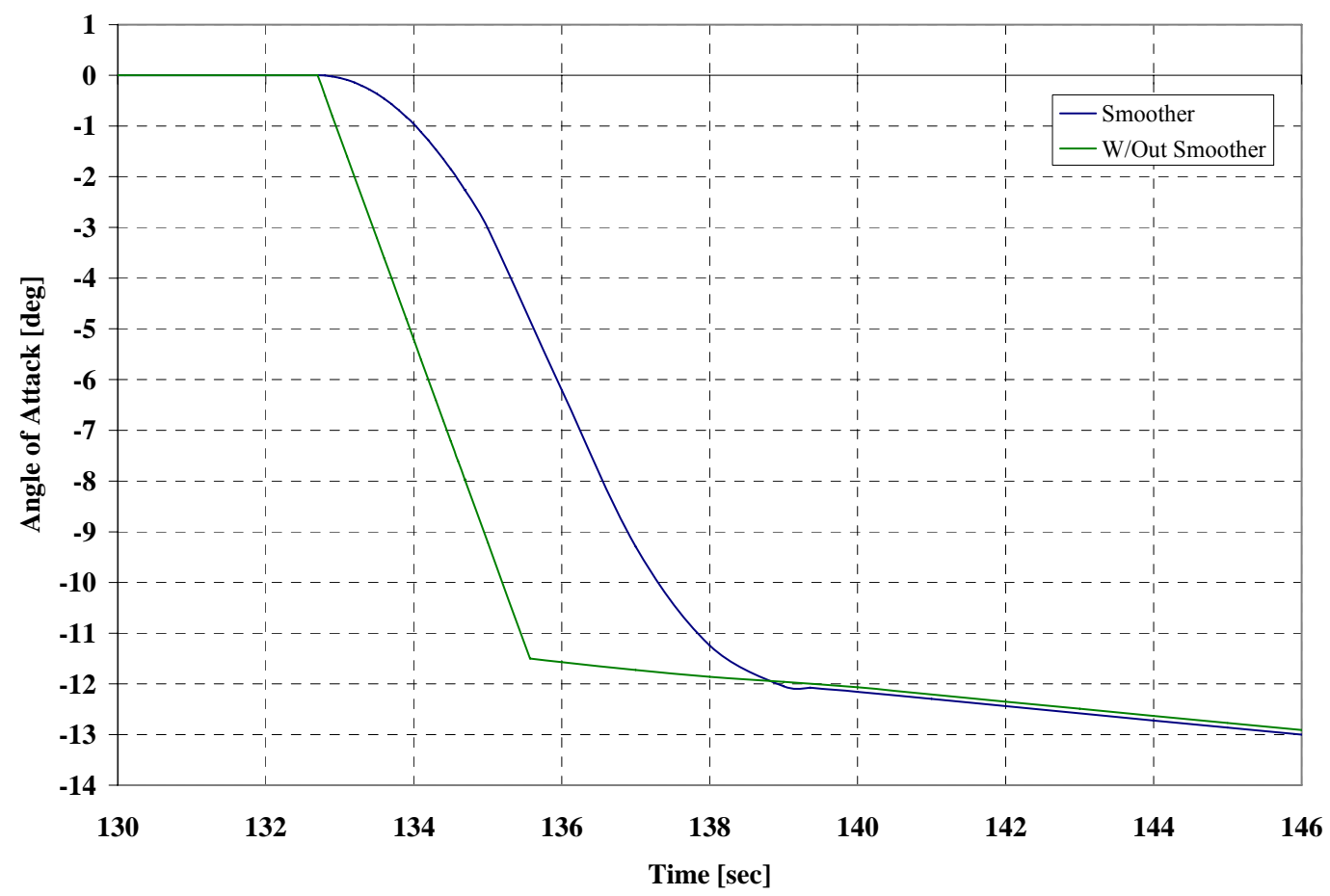

Figure 5. Angle of Attack Profile with Smoother and with Optimized Rate.

As with the roll maneuver, a single angle of attack curve that the trajectory should follow could not be predetermined. Therefore, a family of curves was created for the angle of attack maneuver and implemented in POST. During the phase of the trajectory immediately following this maneuver, angle of attack is an independent parameter that POST varies for the optimized trajectory. The starting angle was the same $\left(0.0^{\circ}\right)$ for each curve produced. The final angle of attack varied from $-9^{\circ}$ to $-15^{\circ}$ in one degree increments. The implementation in POST was carefully constructed so it could choose the correct curve while allowing the angle of attack at the end of the maneuver to be an independent variable.

Like the roll maneuver, the vehicle configuration 6-DOF properties determine the angle of attack curves. The values used in the following calculations are found in Table 2. This is representative of a vehicle similar to an Ares I vehicle and is used as an example. 
Table 2. Angle of Attack Maneuver Calculation Inputs.

\begin{tabular}{|l|l|}
\hline Thrust & $294,000.0 \mathrm{lbf}$ \\
\hline Moment arm $(\mathrm{d})$ & $32.55 \mathrm{ft}$ \\
\hline $\mathrm{I}_{\text {pitch }}\left(\mathrm{I}_{\mathrm{yy}}\right)$ & $12,582,447.0$ slug- $\mathrm{ft}^{2}$ \\
\hline Gimbal Angle & $1.5^{\circ}$ \\
\hline Initial Angle & $0.0^{\circ}$ \\
\hline Initial Angular Velocity & $0.0 \mathrm{deg} / \mathrm{sec}$ \\
\hline Final Angle & $-15^{\circ}:-9^{\circ}\left(1.0^{\circ}\right.$ increments $)$ \\
\hline Final Angular Velocity & $0.0 \mathrm{deg} / \mathrm{sec}$ \\
\hline
\end{tabular}

A sample of the family of angle of attack curves is found in Figure 6.

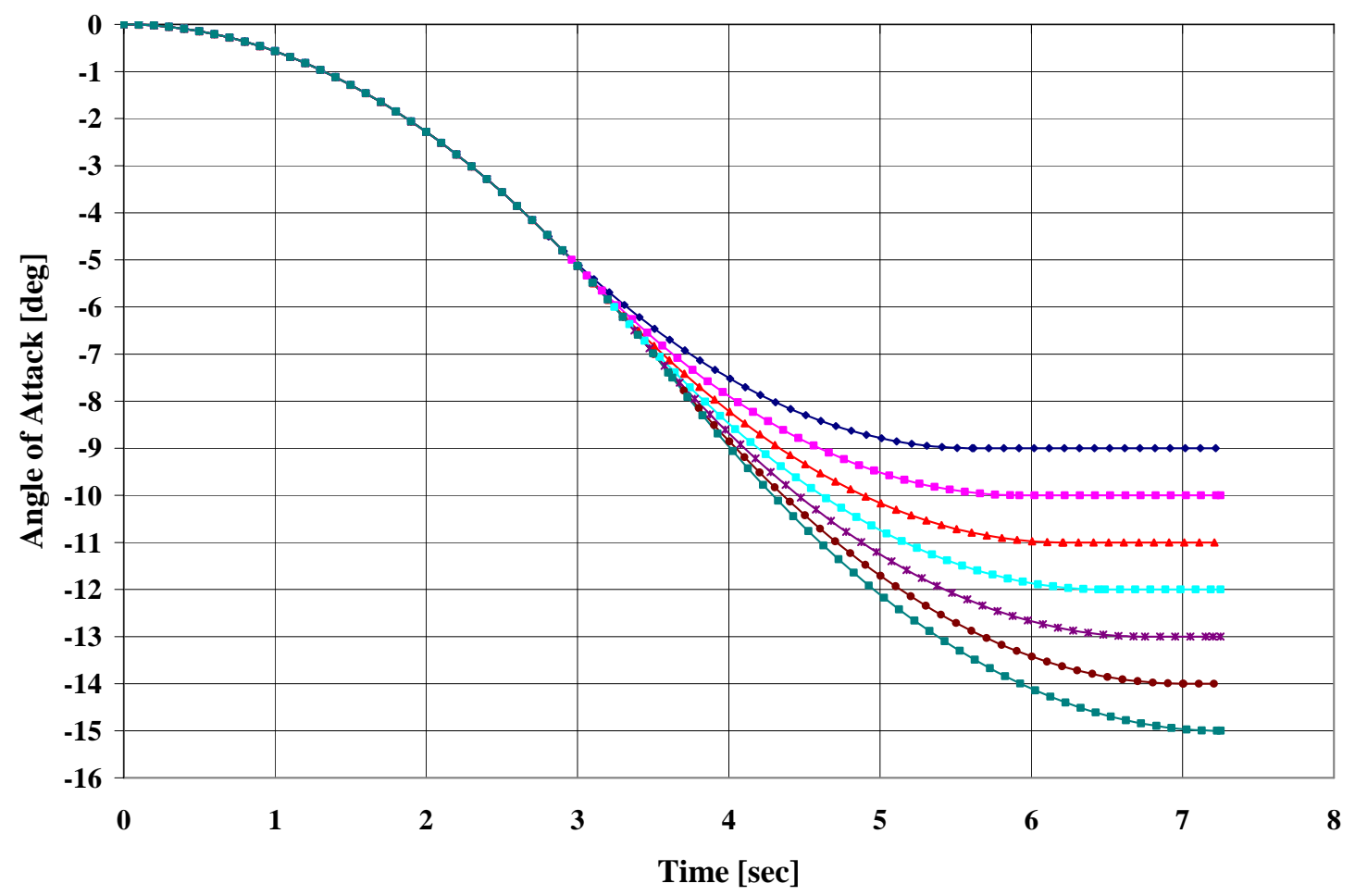

Figure 6. Angle of Attack Maneuver Curves $\left(-9^{\circ}\right.$ to $\left.-15^{\circ}\right)$.

\section{Non-Constant Thrust}

Originally, it was thought that the angle of attack maneuver should begin with the J-2X engine start command. This would cause the thrust to be time dependent due to the start-up transients. Therefore, the angular acceleration in the previous equations would no longer be constant. Equations for non-constant angular acceleration dependent on time were formulated, expanding on the previous formulation. The following method incorporates the angular acceleration based on a cubic expression for thrust with respect to time. These expressions are applicable to maneuvers during any flight regime with non-constant thrust (i.e. during the flight of a solid rocket booster).

The angular acceleration is found in Eq. (10).

$$
\ddot{\theta}=\frac{L \sin \delta}{I_{\text {pitch }}}\left(T_{1} t^{3}+T_{2} t^{2}+T_{3} t+T_{4}\right)=\alpha\left(T_{1} t^{3}+T_{2} t^{2}+T_{3} t+T_{4}\right)
$$

Where $L$ is the moment arm, $\delta$ is the allowed gimbal angle and $I_{\text {pitch }}$ is the inertia around the pitch axis. For the roll maneuver, remove the $\sin \delta$ term from Eq. (10). $T_{1}, T_{2}, T_{3}$, and $T_{4}$ describe the thrust curve for the time period 
that the maneuver occurs. These variables represent the coefficients of a cubic curve fit through the thrust data. Integrate the angular acceleration to yield the angular velocity and angle, Eq. (11) and Eq. (12).

$$
\begin{gathered}
\dot{\theta}=\alpha\left[\frac{1}{4} T_{1} t^{4}+\frac{1}{3} T_{2} t^{3}+\frac{1}{2} T_{3} t^{2}+T_{4} t\right]+c_{1} \\
\theta=\alpha\left[\frac{1}{20} T_{1} t^{5}+\frac{1}{12} T_{2} t^{4}+\frac{1}{6} T_{3} t^{3}+\frac{1}{2} T_{4} t^{2}\right]+c_{1} t+c_{2}
\end{gathered}
$$

As in the constant angular acceleration cases, the following conditions are known: the maneuver start time, the initial pitch angle, the final pitch angle, and the initial and final angular velocities. The continuity constraint on the angular velocity and pitch angle remains imposed at the switch point. Following the steps in Section II, these conditions were sufficient to produce a set of two equations dependent only on switch time and maneuver end time. Equation (13) can be solved mathematically to produce a valid set of switch and end times.

$$
\begin{aligned}
& -\dot{\theta}_{e}-\alpha\left[\frac{1}{4} T_{1} t_{e}^{4}+\frac{1}{3} T_{2} t_{e}^{3}+\frac{1}{2} T_{3} t_{e}^{4}+T_{4} t_{e}\right]+\alpha\left[\frac{1}{2} T_{1} t_{s w}^{4}+\frac{2}{3} T_{2} t_{s w}^{3}+T_{3} t_{s w}^{2}+2 T_{4} t_{s w}\right]+c_{1 s}=0 \\
& -\theta_{e}-\alpha\left[\frac{1}{20} T_{1} t_{e}^{5}+\frac{1}{12} T_{2} t_{e}^{4}+\frac{1}{6} T_{3} t_{e}^{3}+\frac{1}{2} T_{4} t_{e}^{2}\right]+\dot{\theta}_{e}+\alpha t_{e}\left[\frac{1}{4} T_{1} t_{e}^{4}+\frac{1}{3} T_{2} t_{e}^{3}+\frac{1}{2} T_{3} t_{e}^{2}+T_{4} t_{e}\right]+ \\
& \alpha\left[\frac{1}{10} T_{1} t_{s w}^{5}+\frac{1}{6} T_{2} t_{s w}^{4}+\frac{1}{3} T_{3} t_{s w}^{3}+T_{4} t_{s w}^{2}\right]-\dot{\theta}_{e} t_{s w}-\alpha t_{s w}\left[\frac{1}{4} T_{1} t_{e}^{4}+\frac{1}{3} T_{2} t_{e}^{3}+\frac{1}{2} T_{3} t_{e}^{2}+T_{4} t_{e}\right]+c_{1 s} t_{s w}+c_{2 s}=0
\end{aligned}
$$

Once the switch and end times are known, the remaining unknown constants may be calculated. The equations can then be used to calculate the curves as in the previous cases.

\section{Additional Applications}

An additional suggested application of these equations comes from closed-loop dispersion simulations. When the closed-loop guidance system is determining the flight control commands, while running Monte Carlo simulation sets or during flight, an equation can be developed to interact with the flight control system for this flight phase. This equation would be based on the smooth maneuver curve that the optimizer chose for the nominal optimized trajectory, instead of the family of curves. Allowing the flight controller to use the same smooth maneuver as the optimized trajectory will prevent disconnects between the two simulations from occurring. Following the examples in this paper, this equation would represent the angle of attack curve from the POST trajectory, which can be represented accurately by the fifth order polynomial curve fit, (Eq. (14)).

$$
y=a_{5} x^{5}+a_{4} x^{4}+a_{3} x^{3}+a_{2} x^{2}+a_{1} x+a_{0}
$$

The polynomial equation can be adjusted to account for slight changes to the nominal profile, such as a different starting or ending angle (or both). These changes would appear as a consequence of running dispersed trajectories. The initial fifth order equation works best when the starting time is forced to be zero and the y-axis crossing is forced to be the initial angle. The polynomial can be adjusted for small starting angle uncertainties through the constant term, $a_{0}$. The initial starting time of the curve is $0.0 \mathrm{sec}$, when this is substituted in for the time, and the initial angle for the $y$, then $a_{0}$ can be determined.

The end point can also be accounted for in the polynomial adjustment. For the closed-loop guidance implementation, assume that the length of time required to complete the original curve from POST is fixed. If the new final end angle is known, then Eq. (14) can be solved for a new value of $a_{l}$, with this end point. It is recommended only to adjust the $a_{l}$ and $a_{0}$ terms when accounting for uncertainties that affect the initial and final angles. Even though changes in the slope of the curve require a different angular acceleration, there was enough 
margin accounted for in the original calculation to allow small adjustments and still be able to successfully fly the vehicle.

These calculations were performed and compared to the original curves to verify that this method adequately represents the new points as compared to the curve produced by the original smooth maneuver method. This check was done without being incorporated into a simulation. The current Ares I guidance procedure uses a different approach to smoothing, with a fixed limit on the attitude accelerations and rates passed to the flight control. These limits serve the same purpose as a guidance implementation of this smooth maneuver procedure, but do not come as close to matching the POST trajectory design.

\section{Conclusion}

A new approach to smooth trajectory maneuvers has been successfully implemented in the Ares I design analysis cycle process. The method developed here provides a smooth maneuver profile for launch vehicle applications. Smooth profiles that do not demand all of the available control power are important, so that the flight control system is not saturated and in order to minimize overshoot of desired vehicle attitudes. A saturated system can lead to instability of the vehicle, since it can no longer counteract the various torques and perturbations affecting the vehicle. The smooth roll maneuver (for First Stage flight) and smooth angle of attack maneuver (after Upper Stage engine ignition) have successfully removed the gap occurring between the different Ares I trajectory analysis tools.

\section{Acknowledgments}

The authors wish to acknowledge Adam Brinckerhoff who was involved during the preliminary stages of this investigation. Mr. Brinckerhoff was part of the MSFC internship program when these concepts were first being explored. He is currently a graduate student at the Georgia Institute of Technology.

\section{References}

${ }^{1}$ McHenry, R. L., Brand, T. J., Long, A. D., Cockerell, B. F., and Thibodeau III, J. R., "Space Shuttle Ascent Guidance, Navigation and Control," The Journal of the Astronautical Sciences, Vol. XXVII No. 1, 1979, pp. 1, 38.

${ }^{2}$ Powell, R. W., Striepe, S. A., Desai, P.N., and Braun, R.D., "Program To Optimize Simulated Trajectories (POST) Utilization Manual” NASA Langley Research Center., Volume II, Version 5.2, Hampton VA. Oct. 1997. 